\section{Pre-treatment maximum standardized uptake value predicts outcome after frontline therapy in patients with advanced stage follicular lymphoma}

\author{
Paolo Strati, ${ }^{1}$ Mohamed Amin Ahmed, ${ }^{1}$ Nathan H. Fowler, ${ }^{1}$ \\ Loretta J. Nastoupil, ${ }^{1}$ Felipe Samaniego, ${ }^{1}$ Luis E. Fayad, ${ }^{1}$ \\ Fredrick B. Hagemeister, ${ }^{1}$ Jorge E. Romaguera, ${ }^{1}$ Alma Rodriguez, ${ }^{1}$ \\ Michael Wang, ${ }^{1}$ Jason R. Westin, ${ }^{1}$ Chan Cheah, ${ }^{1}$ Mansoor Noorani, ${ }^{1}$ Lei Feng, ${ }^{2}$ \\ Richard E. Davis ${ }^{1}$ and Sattva S. Neelapu ${ }^{1}$ \\ ${ }^{1}$ Department of Lymphoma and Myeloma, The University of Texas MD Anderson Cancer \\ Center and ${ }^{2}$ Department of Biostatistics, The University of Texas MD Anderson Cancer \\ Center, Houston, TX, USA
}

\section{Ferrata Storti Foundation}

Haematologica 2020

Volume 105(7):1907-1913

\section{ABSTRACT}

T he impact of pre-treatment maximum standardized uptake value $\left(S_{U} V_{\max }\right)$ on the outcome of follicular lymphoma (FL) following specific frontline regimens has not been explored. We performed a retrospective analysis of 346 patients with advanced stage follicular lymphoma (FL) without histological evidence of transformation, and analyzed the impact of $\mathrm{SUV}_{\max }$ on outcome after frontline therapy. Fifty-two $(15 \%)$ patients had a $\mathrm{SUV}_{\max }>18$, and a large lymph node $\geq 6 \mathrm{~cm}$ was the only factor associating with $\mathrm{SUV}_{\max }>18$ on multivariate analysis (odds ratio $2.7,95 \%$ confidence interval [CI]: 1.3-5.3, $P=0.006$ ). The complete response rate was significantly lower among patients treated with non-anthracycline-based regimens if $\mathrm{SUV}_{\text {max }}$ was $>18$ ( $45 \%$ vs. $92 \%, P<0.001$ ), but not among patients treated with R-CHOP $(P=1)$. SUV $\mathrm{Sax}_{\max }>18$ was associated with significantly shorter progression-free survival among patients treated with non-anthracycline-based regimens ( 77 months vs. not reached, $P=0.02$ ), but not among patients treated with R-CHOP $(P=0.73)$. $\mathrm{SUV}_{\max }>18$ associated with shorter overall survival (OS) both in patients treated with R-CHOP (8-year OS 70\% vs. $90 \%, P=0.02$ ) and non-anthracycline-based frontline regimens (8-year OS $50 \%$ vs. $85 \%, P=0.001$ ). In conclusion, pre-treatment PET scan has prognostic and predictive value in patients with advanced stage FL receiving frontline treatment.

\section{Introduction}

Despite its indolent biology, follicular lymphoma (FL) can be fluorodeoxyglucose (FDG) avid on positron emission tomography (PET). A wide range of inter- and intra-patient degree of FDG avidity has been reported, with a maximum standardized uptake $\left(\mathrm{SUV}_{\max }\right)$ value ranging between 3 and $40 \cdot \cdot^{1-4}$ PET scan is more sensitive and specific than standard computed tomography (CT) scan in identifying nodal and extra-nodal disease, altering stage assignation in 10-31\% of patients with FL, and determining a treatment plan revision based on upstaging (I-II to III-IV) in 34$45 \%$ of cases. . $^{5-10}$ PET-based imaging is also an effective tool for early detection of FL transformation, incremental threshold of $\mathrm{SUV}_{\max }$ values associating with increasing test specificity. ${ }^{11-14}$ False positivity is still possible, though, and histological confirmation through tissue biopsy is recommended. ${ }^{15,16}$

Despite its beneficial effect on staging reassignment and histological classification, the prognostic role of PET-based imaging at time of diagnosis remains unclear, with conflicting data published in the literature with regards to its impact on the Follicular Lymphoma International Prognostic Index (FLIPI) determination. ${ }^{6,8,17}$ In addition, while multiple studies have investigated the association between a posttreatment PET scan and the risk of relapse, limited data are available regarding the predictive role of pre-treatment PET data in the frontline setting. ${ }^{18-22}$ We provide a
SATTVA S. NEELAPU

sneelapu@mdanderson.org

Received: June 28, 2019.

Accepted: October 4, 2019.

Pre-published: October 10, 2019.

doi:10.3324/haematol.2019.230649

Check the online version for the most updated information on this article, online supplements, and information on authorship \& disclosures: www.haematologica.org/content/105/7/1907

(C)2020 Ferrata Storti Foundation

Material published in Haematologica is covered by copyright. All rights are reserved to the Ferrata Storti Foundation. Use of published material is allowed under the following terms and conditions:

https://creativecommons.org/licenses/by-nc/4.0/legalcode. Copies of published material are allowed for personal or internal use. Sharing published material for non-commercial purposes is subject to the following conditions:

https://creativecommons.org/licenses/by-nc/4.0/legalcode, sect. 3. Reproducing and sharing published material for commercial purposes is not allowed without permission in writing from the publisher.

\section{Correspondence:}


retrospective analysis of 346 patients with advanced stage FL, in whom a PET-CT scan was performed prior to initiation of therapy, and analyze the impact of SUV $\max$ on the quality of response and outcomes after frontline therapy.

\section{Methods}

\section{Patient selection}

This is a retrospective analysis of patients with stage III or IV FL, grades I, II, or IIIA, receiving frontline treatment at the $\mathrm{MD}$ Anderson Cancer Center (MDACC) between August, 2001 and April, 2014, and who had a pre-treatment PET-CT scan performed. Patients with histological diagnosis of FL grade IIIB or concurrent diffuse large B-cell lymphoma (DLBCL) were excluded.

The clinical and laboratory features were confirmed by review of the medical records. Frontline therapy was administered according to the previously described schedule. ${ }^{23-27}$ The FLIPI and FLIPI-2 scores were calculated as previously described. ${ }^{28,29}$ Lugano classification was used to define complete response..$^{30,31}$ The study was approved by the Institutional Review Board of the MDACC and conducted in accordance with our institutional guidelines and the principles of the Declaration of Helsinki.

\section{PET scan and SUV max $_{\text {threshold selection }}$}

Baseline PET-CT scans were obtained at MDACC before initiation of frontline therapy. After patients had fasted for at least 4-6 hours, blood glucose was measured and confirmed to be $<140 \mathrm{mg} / \mathrm{dL}$ ( $<200 \mathrm{mg} / \mathrm{dL}$ for patients with diabetes) before injection of 333-407 MBq (9-11 mCi) of $\left[{ }^{18} \mathrm{~F}\right] \mathrm{FDG}$. Emission scans were acquired at 2-3 minutes per field of view in the threedimensional mode after a 60 -minute uptake time ( \pm 10 minutes). CT non-contrast images were acquired in helical mode with $3.75-\mathrm{mm}$ slices from the skull base through the midthigh. Commercially available iterative algorithms were used for image reconstruction. PET images were collected and transferred to commercially available software (MIMVista version 6.4.9; MIMVista Corporation, Cleveland, OH). SUV $\max$ was calculated as previously described. ${ }^{32}$ All reports of pre-treatment scans and of scans performed to assess response to frontline therapy were reviewed by an oncologist with expertise in lymphoma.

Analyzing multiple single unit increments of $\mathrm{SUV}_{\max }$ among all 346 patients included in the study, 18 showed the strongest association with progression-free survival (PFS) (hazard ratio [HR] 1.5, 95\% confidence interval [CI]: 0.95-2.3, $P=0.08)$, and was selected as cut-off for further analysis (Online Supplementary Table S1).

\section{Statistical methods}

Association with categorical variables was evaluated using $\chi^{2}$ or Fisher exact tests, or the Mann-Whitney test, as appropriate, and logistic regression was used for multivariate analysis. Only factors significant $(P$-value $<0.05)$ on univariate analysis were included in multivariate models. PFS was defined as the time from the start of therapy to progression of disease, death, or last follow-up (whichever occurred first). Overall survival (OS) was defined as the time from the start of therapy to death or last follow-up. PFS and OS were calculated for all patients in the study and for subgroups of patients using Kaplan-Meier estimates and were compared between subgroups using the log-rank test. Multivariable Cox regression analysis was performed to assess the associations between patient characteristics and PFS or OS. A $P$-value of $<0.05$ (two-tailed) was considered statistically significant. Statistical analyses were completed using SPSS 21.

\section{Results}

\section{Patient baseline characteristics}

Three-hundred and forty-six patients were included in the study, median SUV $_{\max }$ was 11 (range: $1.5-42$ ), and 52 $(15 \%)$ patients had a $\mathrm{SUV}_{\max }>18$. All 52 patients with $\mathrm{SUV}_{\max }>18$ had a biopsy of the most FDG-avid lymph node, and no histological evidence of transformation was observed. Baseline characteristics are shown in Table 1. On univariate analysis, factors associated with $\mathrm{SUV}_{\max }>18$ were male sex $(67 \%$ vs. $52 \%, P=0.05)$, elevated $\beta 2$-microglobulin ( $65 \%$ vs. $47 \%, P=0.02)$, elevated lactate dehydrogenase $(\mathrm{LDH})(37 \%$ vs. $13 \%, P<0.001)$, presence of B symptoms ( $35 \%$ vs. $14 \%, P=0.01)$, and a large lymph node $>6 \mathrm{~cm}(64 \%$ vs. $30 \%, P<0.001)$ (Table 1$)$. On multivariate analysis, a large lymph node $\geq 6 \mathrm{~cm}$ was the only factor maintaining its association with $\mathrm{SUV}_{\max }>18$ (odds ratio [OR] 2.7, 95\% CI: 1.3-5.3, $P=0.006$ )(Table 2 ).

Response to frontline therapy: complete response (CR) One-hundred and fifty-one (44\%) patients were treated with frontline rituximab, cyclophosphamide, doxorubicin, vincristine and prednisone (R-CHOP), and 195 (56\%) with other therapies, including rituximab and bendamustine (BR) in $55(16 \%)$ patients, rituximab and lenalidomide $\left(\mathrm{R}^{2}\right)$ in $63(18 \%)$, rituximab, fludarabine, mitoxantrone, and dexamethasone (R-FND) in $24(7 \%)$, and single agent rituximab in $53(15 \%)$ patients. Two-hundred and thirtytwo $(65 \%)$ patients received maintenance rituximab and $114(33 \%)$ were observed after completion of frontline therapy. While no differences in the use of maintenance therapy were observed between the two groups (75\% vs. $67 \%, P=0.20$ ), a significantly higher proportion of patients with baseline pre-treatment $\mathrm{SUV}_{\max }>18$ were treated with $\mathrm{R}-\mathrm{CHOP}$ versus non-anthracycline-based regimens (75\% vs. $38 \%, P<0.001)$, so subsequent results were stratified by treatment arm.

Among 342 patients evaluable for response, 305 (89\%) achieved CR; the CR rate was $91 \%$ for patients with $\mathrm{SUV}_{\max }<18$ and $80 \%$ for patients with $\mathrm{SUV}_{\max }>18$ $(P=0.03)$. No association between $\mathrm{SUV}_{\max }$ and $\mathrm{CR}$ rate was observed among patients treated with R-CHOP (89\% for $\mathrm{SUV}_{\text {max }}>18$ vs. $88 \%$ for $\mathrm{SUV}_{\max }<18, P=1$ ). However, SUV $_{\max }>18$ significantly associated with a lower CR rate among patients treated with other frontline regimens $\left(45 \%\right.$ for $\mathrm{SUV}_{\max }>18$ vs. $92 \%$ for $\left.\mathrm{SUV}_{\max }<18\right)(P<0.001)$ (Figure 1). After excluding patients treated with single agent rituximab a trend for a lower CR rate among patients treated with other frontline regimens and SUV $>18$ was observed ( $80 \%$ vs. $94 \%, P=0.17$ ); of interest, in this group, only five patients with $\mathrm{SUV}_{\max }>18$ were evaluable for response, likely limiting achievement of statistical significance.

\section{Progression-free survival (PFS) and PFS24}

After a median follow-up of 94 months (95\% CI: 88-100 months), median PFS was not reached, and a trend for decreased PFS was observed in patients with baseline SUV $_{\max }>18$ compared to patients with baseline $<18$ (114 months vs. not reached, $P=0.08)$. Baseline $\geq 18$ did not associate with shorter median PFS among patients treated with frontline R-CHOP (114 months vs. 144 months, $P=0.73)$, but it did associate with significantly shorter PFS among patients treated with other frontline regimens (77 months vs. not reached, $P=0.02$ )(Figure 2 ). After excluding 
patients treated with single agent rituximab, a trend for shorter PFS among patients treated with other frontline regimens and baseline $\mathrm{SUV}_{\max }>18$ was observed (77 months $v s$. not reached, $P=0.17$ ); of interest, in this group, only seven patients with baseline $\mathrm{SUV}_{\max }>18$ were evalu- able for survival, likely limiting achievement of statistical significance.

Use of maintenance rituximab associated with a significantly longer PFS (not reached vs. 84 months, $P<0.001$ ); the association between use of maintenance rituximab

Table 1. Patient baseline characteristics and association with pre-treatment maximum standardized uptake value $\left(\mathrm{SUV}_{\max }\right)>18$.

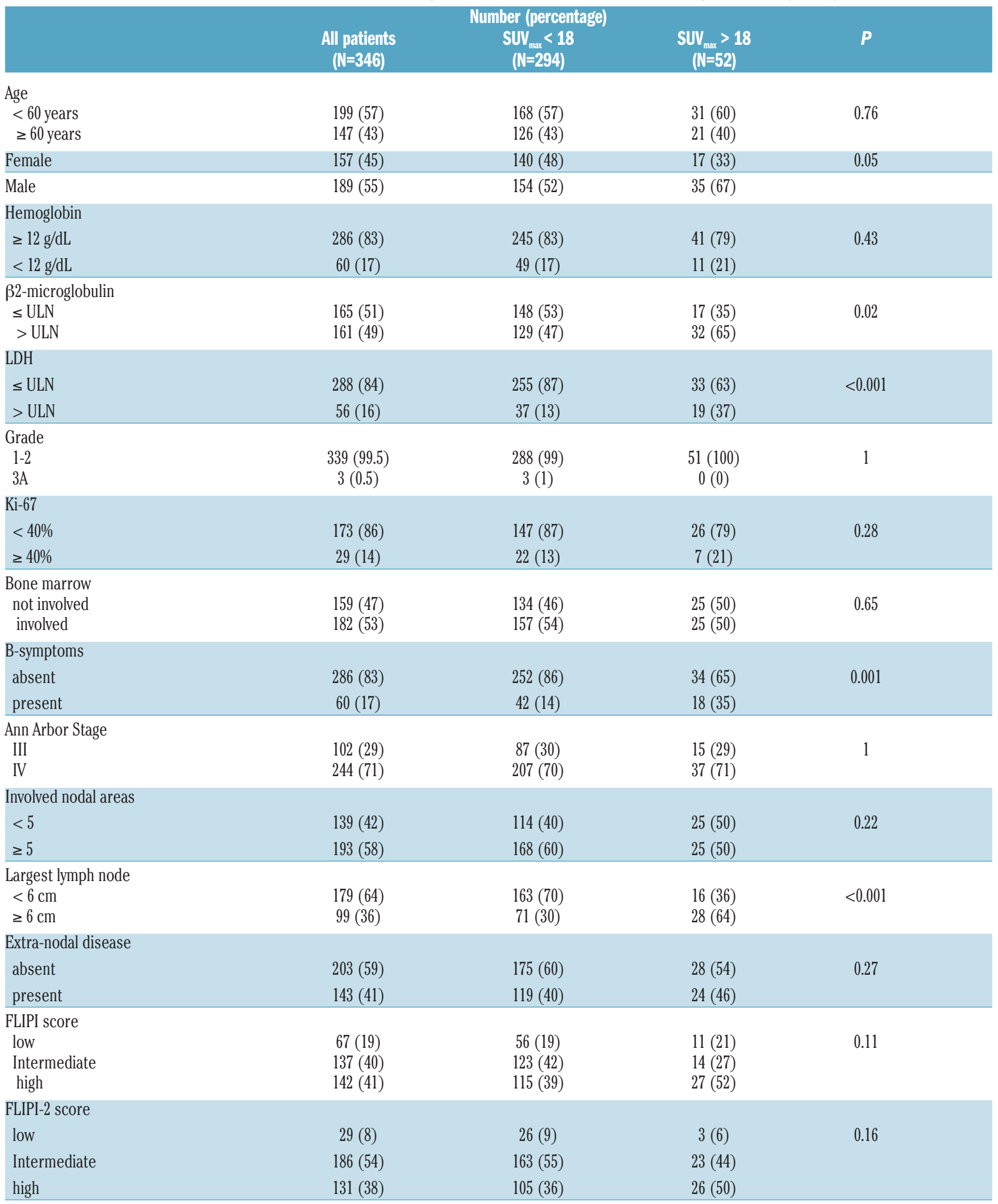

LDH: lactate dehydrogenase; ULN: upper limit of normal; FLIPI: follicular lymphoma international prognostic index; $\mathrm{SUV}_{\max }$ : maximum standardized uptake value. 


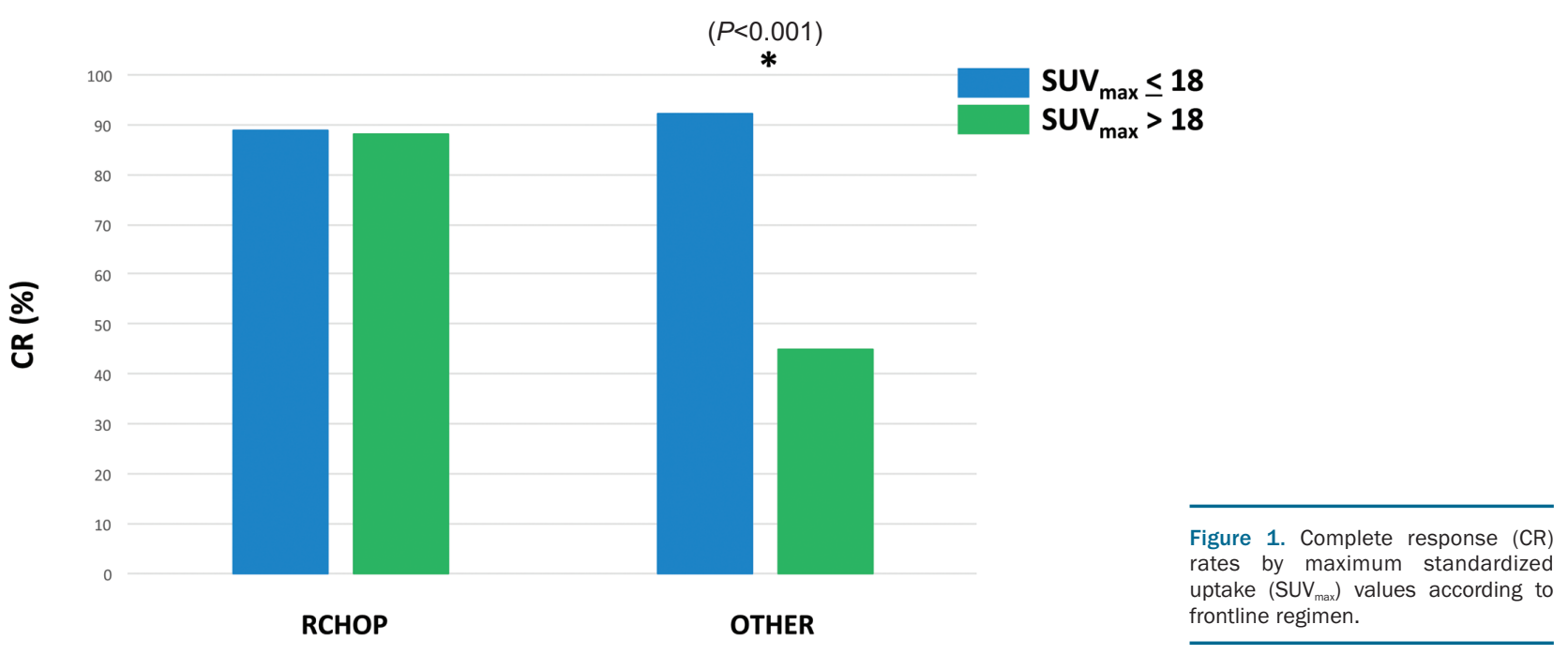

and prolonged PFS was maintained also after adjusting for pre-treatment $\mathrm{SUV}_{\max }>18$ (HR 2, 95\% CI: 1.3-2.9, $P<0.001)$. Further subgroup analysis, to assess the effect of maintenance rituximab after specific regimens according to pre-treatment $\mathrm{SUV}_{\max }>18$ could not be performed, because of small population samples.

Excluding 11 patients who did not progress and were lost to follow-up within 24 months, $67(20 \%)$ patients had a PFS of less than 24 months: PFS $<24$ months was observed in $51(18 \%)$ patients with baseline $\mathrm{SUV}_{\max }<18$, and $16(34 \%)$ of patients with baseline $\mathrm{SUV}_{\max }>18$ $(P=0.02)$. $S U V_{\max }>18$ did not associate with a significantly higher rate of PFS $<24$ months among patients treated with R-CHOP ( $32 \%$ vs. $24 \%, P=0.39)$, but it did associate with a higher rate of PFS $<24$ months among patients treated with other regimens ( $40 \%$ vs. $14 \%, P=0.05)$. After excluding patients treated with single agent rituximab, a trend for higher PFS24 rate among patients treated with other frontline regimens and $\mathrm{SUV}_{\max }>18$ was observed ( $20 \%$ vs. $11 \%, P=0.45)$; of interest, only five patients with $\mathrm{SUV}_{\max }>18$ were evaluable for PFS24, likely limiting achievement of statistical significance.

\section{Overall survival (OS) and risk of transformation}

After a median follow-up of 94 months (95\% CI: 88-100 months), median OS has not been reached, and it was significantly shorter among patients with $\mathrm{SUV}_{\max }>18$ (8-year OS $65 \%$ vs. $89 \%, P=0.001)$. $S U_{\max }>18$ associated with shorter OS both in patients treated with frontline RCHOP (8-year OS $70 \%$ vs. $90 \%, P=0.02$ ) and in patients treated with other frontline regimens (8-year OS $50 \%$ vs. $85 \%, P=0.001$ )(Figure 3). A trend for longer OS was observed among patients with $\mathrm{SUV}_{\max }>18$ when comparing treatment with frontline R-CHOP to other frontline treatments (8-year OS $70 \%$ vs. $50 \%, P=0.15$ ). The association between $\mathrm{SUV}_{\max }>18$ and OS was maintained also on multivariate analysis including either FLIPI score (HR 2.6, $95 \%$ CI: $1.5-4.6, P=0.001)$ or FLIPI-2 score (HR $2.2,95 \%$ CI: 1.3-3.9, $P=0.006$ ).

At the most recent follow-up, $18(5 \%)$ patients have progressed with histological evidence of transformation to large B-cell lymphoma, after a median time of 23 months (range: 5-139 months). Twelve transformations (4\%) occurred among patients with baseline $\mathrm{SUV}_{\text {max }}<18$, and six $(11 \%)$ among patients with baseline $\mathrm{SUV}_{\max }>18$ $(P=0.04)$. Of these 18 patients, 11 had received frontline $\mathrm{R}$ CHOP (of whom six had baseline $\mathrm{SUV}_{\max }>18$ ) and seven other frontline regimens (none of whom had baseline $S U V_{\text {max }}>18$ ). After excluding patients treated with single agent rituximab, a statistically significant shorter OS among patients treated with other frontline regimens and $S \mathrm{VV}_{\max }>18$ was observed (86 months vs. not reached, $P=0.05)$.

\section{Discussion}

Recent guidelines recommend the use of PET-CT in FL for initial staging, evaluation of potential transformation, and at time of response assessment. ${ }^{31,33}$ However, the impact of baseline PET-based imaging on the outcome following specific frontline treatment has not been previously explored.

In our analysis, an $\mathrm{SUV}_{\max }$ cut-off of 18 was identified as clinically significant, showing the strongest association with PFS. SUV max $_{\text {ax }}$ cut-offs of 10, 14 and 17 have been proposed in previous retrospective studies to identify patients with FL at higher risk of transformation to DLBCL, ${ }^{11,12,14}$ with OR for transformation of 1.25 for each increase in unit of SUV $\mathrm{Smx}_{\text {max }}{ }^{10}$ To this regard, patients with histological evidence of FL grade IIIB or DLBCL were excluded from this study. Although tumor heterogeneity may have caused a false-negative result, elevated $\mathrm{SUV}_{\max }$ in the absence of histological evidence of transformation may reflect a more aggressive biology, associating with a worse outcome, as already shown in other forms of low grade B-cell lymphomas. ${ }^{34}$ It is interesting to note that in our study the only factor significantly associated with $S_{U} V_{\text {max }}$ $>18$ was the lymph node size $\geq 6 \mathrm{~cm}$ and that more transformations occurred in patients with pre-treatment $S V_{\max }$ $>18(11 \%$ vs. $4 \%, P=0.04)$. While a core needle biopsy of a large lymph node may miss a diagnosis of DLBCL, the large tumor size may also simply be a surrogate marker of accelerated biology in the absence of transformation. Prospective studies, employing excisional biopsy, as opposed to core biopsy, may shed light on this question.

In our study, patients with $\mathrm{SUV}_{\max }>18$ were more frequently treated with R-CHOP, compared to other thera- 


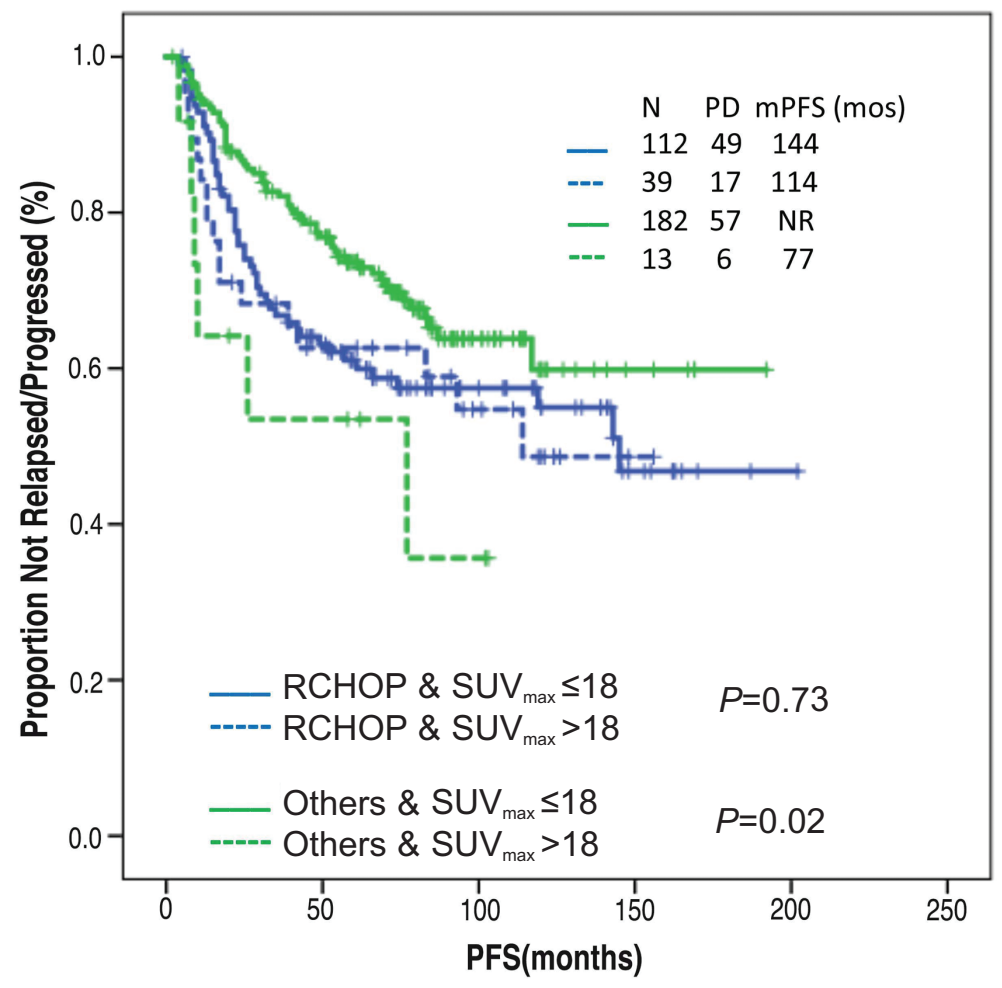

Figure 2. Association between progression-free survival (PFS) and maximum standardized uptake $\left(\mathrm{SUV}_{\max }\right)$ separated by frontline treatment arm.

Table 2. Multivariate analysis of baseline characteristics associated with pre-treatment maximum standardized uptake value $\left(\mathrm{SUV}_{\max }\right)>18$

\begin{tabular}{|c|c|c|c|}
\hline & Total (N=263) & $\begin{array}{l}\text { nber (percent } \\
\text { OR [95\% Cl] }\end{array}$ & P-multi \\
\hline Female & 123 & $1.9[0.9-3.8]$ & 0.08 \\
\hline Male & 140 & & \\
\hline \multicolumn{4}{|c|}{$\beta 2$-microglobulin } \\
\hline$\leq \mathrm{ULN}$ & 135 & $1.3[0.6-2.7]$ & 0.56 \\
\hline$>$ ULN & 128 & & \\
\hline \multicolumn{4}{|l|}{ LDH } \\
\hline$\leq \mathrm{ULN}$ & 218 & $2[0.9-4.1]$ & 0.08 \\
\hline$>$ ULN & 45 & & \\
\hline \multicolumn{4}{|c|}{ B-symptoms } \\
\hline absent & 214 & & \\
\hline present & 49 & $1.5[0.7-3.1]$ & 0.26 \\
\hline \multicolumn{4}{|c|}{ Largest lymph node } \\
\hline$<6 \mathrm{~cm}$ & 169 & $2.7[1.3-5.3]$ & 0.006 \\
\hline$\geq 6 \mathrm{~cm}$ & 94 & & \\
\hline
\end{tabular}

Overall, 263 of 346 patients had no missing values for all five variables, and were included in the model: 42 with SUV $\max >18$ and 221 with SUVmax $\leq 18$. LDH: lactate dehydrogenase; $\mathrm{ULN}$ : upper limit of normal; $\mathrm{SUV}_{\max }$ : maximum standardized uptake value.

pies (including BR, R-FND, $\mathrm{R}^{2}$ and single agent rituximab). Abou-Nassar et al. had previously reported similar findings, likely reflecting the treating physician's concern for occult transformation, and subsequent need for anthracycline-based therapy. ${ }^{17} \mathrm{We}$ acknowledge as a potential bias of our study that patients with SUV $_{\max }>18$ treated with other therapies may have not been fit for R-CHOP, based on variables not analyzed in this study, such as comorbidities, reflecting a clinician's therapeutic bias, and therefore more likely to experience a dismal outcome.

Of interest, in our study, the association between $\mathrm{SUV}_{\max }$ $>18$ and lower CR rate, observed for the whole popula- tion, was lost among patients treated with R-CHOP. In the rituximab era, the use of anthracycline-based regimens, such as R-CHOP, has significantly improved the outcome of patients with previously untreated transformed FL, resulting in response rates and survival similar to what has been observed in de novo DLBCL. ${ }^{35-38}$ Our results indicate that patients with more aggressive forms of FL, as suggested by pre-treatment $\mathrm{SUV}_{\max }>18$, may benefit from anthracycline-based therapies as the CR rates were significantly lower with alternative regimens. This is further supported by the fact that in our analysis the lower PFS associated with $\mathrm{SUV}_{\max }>18$ (including high rates of progressions 


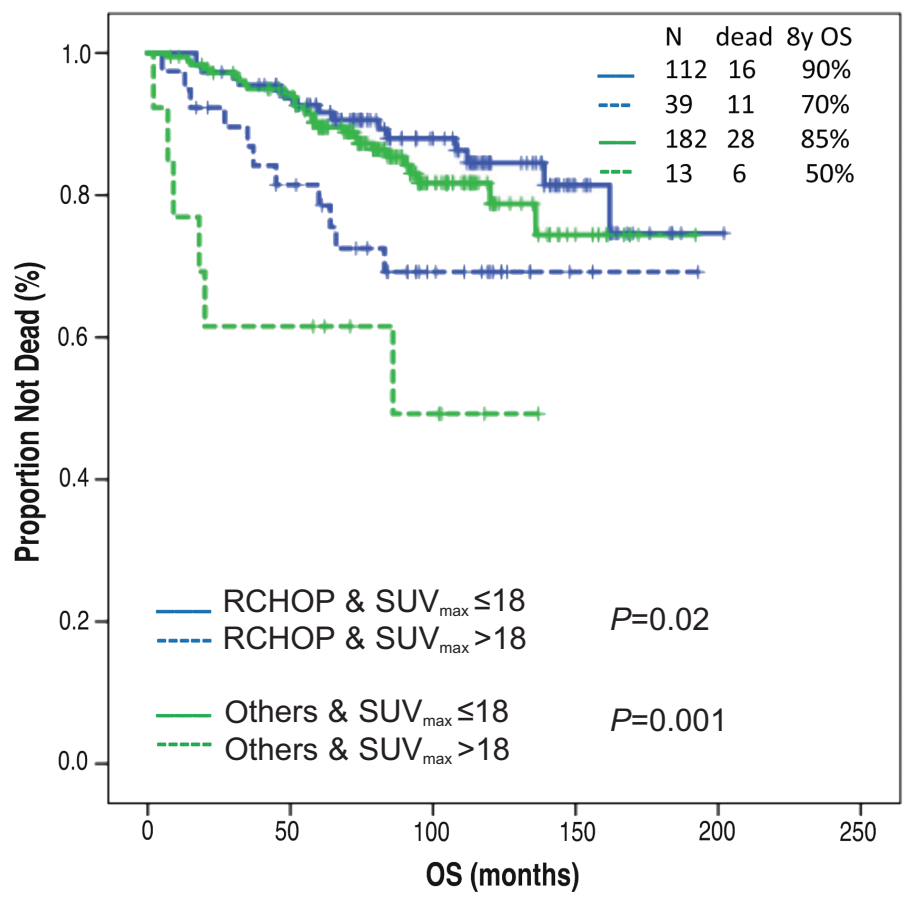

Figure 3. Association between overall survival (OS) and maximum standardized uptake $\left(S U V_{\max }\right)$ separated by frontline treatment arm.

within 24 months from treatment initiation) was overcome by the use of frontline R-CHOP. These novel and clinically relevant findings highlight the need to examine the impact of pre-treatment SUV $\mathrm{max}_{\max }$ on outcomes in recently completed randomized phase 3 trials in FL comparing different frontline regimens such as R-CHOP, BR, and/or $\mathrm{R}^{2} \cdot{ }^{24,25,39}$ It is important to note that, as only three patients with FL grade IIIA were included in this study, these results can apply only to patients with grades I-II FL.

Finally, despite the beneficial effect of R-CHOP on CR rate and PFS of patients with $\mathrm{SUV}_{\max }>18$, subsequent transformations were still observed among patients treated with this regimen. However, this was not unexpected as not all patients with occult or histologically proven transformed FL achieve durable remissions with R-CHOP. Recurrent disease with transformation after R-CHOP can be salvaged with high-dose chemotherapy with autologous stem cell transplantation and/or chimeric antigen receptor T-cell therapy. ${ }^{40-44}$ While patients with transformation at the time of relapse who are anthracycline-naive can be salvaged with R-CHOP, the superior OS observed in patients treated with frontline R-CHOP compared with other regimens within the sub-group of patients with $\mathrm{SUV}_{\max }>18$ (8- year OS $70 \%$ vs. $50 \%, P=0.15$ ) raises the possibility that upfront treatment with anthracyclinebased regimen may lead to better outcomes in such patients. On the other hand, relapses occurring in patients with high $\mathrm{SUV}_{\max }$ after frontline R-CHOP may have a more aggressive biology, including transformation, explaining the difference in $\mathrm{OS}$ according to $\mathrm{SUV}_{\max }$ observed in patients treated with this regimen.

We acknowledge that there are some limitations due to the retrospective nature of this study and the well-known variability and limited reproducibility of SUV measurements. ${ }^{45}$ For example, consistent criteria may not have been used to select patients in whom to perform a tissue biopsy for exclusion of transformation, and therefore patients with concurrent undiagnosed FL grade IIIB and/or DLBCL may have been included in the study. There was also likely variability in dose-intensity of the chemotherapy between patients. The total metabolic tumor volume (TMTV) was not calculated in this analysis, and the latter has been shown to predict the outcome after frontline therapy in patients with high-burden FL. ${ }^{46}$ Upon further validation, TMTV and other PET-based functional parameters, such as total lesion glycolysis, may in the future provide a more standardized approach to assess the prognostic value of pre-treatment PET in patients with FL.

Nevertheless, the significant decrease in the CR rate, $\mathrm{PFS}$, and OS observed in patients with $\mathrm{SUV}_{\max }>18$ in our study suggest that a pre-treatment PET scan has a prognostic and predictive value in patients with advanced stage FL receiving frontline treatment and prospective randomized trials are warranted to investigate this further.

\section{Funding}

This research is supported in part by the MD Anderson Cancer Center Support Grant P30 CA016672.

\section{References}

1. Jerusalem G, Beguin Y, Najjar F, Hustinx R, Fassotte MF, Rigo P, et al. Positron emission tomography (PET) with 18F-fluorodeoxyglucose (18F-FDG) for the staging of low-grade non-Hodgkin's lymphoma (NHL). Ann Oncol. 2001;12(6):825-830.
2. Karam M, Novak L, Cyriac J, Ali A, Nazeer $\mathrm{T}$, Nugent F. Role of fluorine-18 fluorodeoxyglucose positron emission tomography scan in the evaluation and follow-up of patients with low-grade lymphomas. Cancer. 2006;107(1):175-183.

3. Wohrer S, Jaeger U, Kletter K, et al. 18F-fluoro-deoxy-glucose positron emission tomography (18F-FDG-PET) visualizes follicular lymphoma irrespective of grading. Ann Oncol. 2006;17(5):780-784.

4. Elstrom R, Guan L, Baker G, et al. Utility of FDG-PET scanning in lymphoma by WHO classification. Blood. 2003;101(10):38753876.

5. Wirth A, Foo M, Seymour JF, Macmanus MP, 
Hicks RJ. Impact of [18f] fluorodeoxyglucose positron emission tomography on staging and management of early-stage follicular non-hodgkin lymphoma. Int J Radiat Oncol Biol Phys. 2008;71(1):213-219.

6. Luminari S, Biasoli I, Arcaini L, et al. The use of FDG-PET in the initial staging of 142 patients with follicular lymphoma: a retrospective study from the FOLL05 randomized trial of the Fondazione Italiana Linfomi. Ann Oncol. 2013;24(8):2108-2112.

7. Janikova A, Bolcak K, Pavlik T, Mayer J, Kral $Z$. Value of [18F]fluorodeoxyglucose positron emission tomography in the management of follicular lymphoma: the end of a dilemma? Clin Lymphoma Myeloma. 2008;8(5):287-293

8. Le Dortz L, De Guibert S, Bayat S, et al. Diagnostic and prognostic impact of 18FFDG PET/CT in follicular lymphoma. Eur J Nucl Med Mol Imaging. 2010;37(12):23072314.

9. Chen YK, Yeh CL, Tsui CC, Liang JA, Chen $\mathrm{JH}$, Kao CH. F-18 FDG PET for evaluation of bone marrow involvement in non-Hodgkin lymphoma: a meta-analysis. Clin Nucl Med. 2011 Jul;36(7):553-559.

10. Smith SD, Redman M, Dunleavy K. FDG PET-CT in follicular lymphoma: a casebased evidence review. Blood. 2015; 125(7):1078-1082.

11. Schoder H, Noy A, Gonen M, et al. Intensity of 18fluorodeoxyglucose uptake in positron emission tomography distinguishes between indolent and aggressive nonHodgkin's lymphoma. J Clin Oncol. 2005;23(21):4643-4651.

12. Bodet-Milin C, Kraeber-Bodere F, Moreau P, Campion L, Dupas B, Le Gouill S. Investigation of FDG-PET/CT imaging to guide biopsies in the detection of histological transformation of indolent lymphoma. Haematologica. 2008;93(3):471-472.

13. Noy A, Schoder H, Gonen M, et al. The majority of transformed lymphomas have high standardized uptake values (SUVs) on positron emission tomography (PET) scanning similar to diffuse large B-cell lymphoma (DLBCL). Ann Oncol. 2009; 20(3):508-512

14. Karam M, Feustel PJ, Vera CD, Nazeer T. Features of large cell transformation of indolent lymphomas as observed on sequential PET/CT. Nucl Med Commun. 2011;32(3): 177-185.

15. Cook GJ, Fogelman I, Maisey MN. Normal physiological and benign pathological variants of 18-fluoro-2-deoxyglucose positronemission tomography scanning: potential for error in interpretation. Semin Nucl Med. 1996;26(4):308-314

16. Hofman MS, Hicks RJ. Imaging in follicular NHL. Best Pract Res Clin Haematol. 2011;24(2):165-177

17. Abou-Nassar KE, Vanderplas A, Friedberg JW, et al. Patterns of use of 18-fluoro-2deoxy-D-glucose positron emission tomography for initial staging of grade 1-2 follicular lymphoma and its impact on initial treatment strategy in the National Comprehensive Cancer Network NonHodgkin Lymphoma Outcomes database. Leuk Lymphoma. 2013;54(10):2155-2162.

18. Salles G, Seymour JF, Offner F, et al. Rituximab maintenance for 2 years in patients with high tumour burden follicular lymphoma responding to rituximab plus chemotherapy (PRIMA): a phase 3, randomised controlled trial. Lancet. 2011; 377(9759):42-51

19. Luminari S, Biasoli I, Versari A, et al. The prognostic role of post-induction FDG-PET in patients with follicular lymphoma: a subset analysis from the FOLL05 trial of the Fondazione Italiana Linfomi (FIL). Ann Oncol. 2014;25(2):442-447.

20. Trotman J, Fournier M, Lamy T, et al. Positron emission tomography-computed tomography (PET-CT) after induction therapy is highly predictive of patient outcome in follicular lymphoma: analysis of PET-CT in a subset of PRIMA trial participants. J Clin Oncol. 2011;29(23):3194-3200.

21. Dupuis J, Berriolo-Riedinger A, Julian A, et al. Impact of [(18)F]fluorodeoxyglucose positron emission tomography response evaluation in patients with high-tumor burden follicular lymphoma treated with immunochemotherapy: a prospective study from the Groupe d'Etudes des Lymphomes de l'Adulte and GOELAMS. J Clin Oncol. 2012;30(35):4317-4322.

22. Trotman J, Barrington SF, Belada D, et al Prognostic value of end-of-induction PET response after first-line immunochemotherapy for follicular lymphoma (GALLIUM): secondary analysis of a randomised, phase 3 trial. Lancet Oncol. 2018;19(11):1530-1542.

23. Flinn IW, van der Jagt R, Kahl BS, et al. Randomized trial of bendamustine-rituximab or R-CHOP/R-CVP in first-line treatment of indolent NHL or MCL: the BRIGHT study. Blood. 2014;123(19):2944 2952.

24. Rummel MJ, Niederle N, Maschmeyer G, et al. Bendamustine plus rituximab versus $\mathrm{CHOP}$ plus rituximab as first-line treatment for patients with indolent and mantle-cell lymphomas: an open-label, multicentre, randomised, phase 3 non-inferiority trial. Lancet. 2013;381(9873):1203-1210.

25. Morschhauser F, Fowler NH, Feugier P, et al. Rituximab plus Lenalidomide in Advanced Untreated Follicular Lymphoma. N Engl J Med. 2018;379(10):934-947.

26. Nastoupil LJ, McLaughlin P, Feng L, et al. High ten-year remission rates following rituximab, fludarabine, mitoxantrone and dexamethasone (R-FND) with interferon maintenance in indolent lymphoma: Results of a randomized Study. Br J Haematol. 2017;177 (2):263-270

27. Martinelli G, Schmitz SF, Utiger U, $t$ al. Long-term follow-up of patients with follicular lymphoma receiving single-agent rituximab at two different schedules in trial SAKK 35/98. J Clin Oncol. 2010;28(29): 4480-4484

28. Solal-Celigny P, Roy P, Colombat P, et al. Follicular lymphoma international prognostic index. Blood. 2004;104(5):1258-1265

29. Federico M, Bellei M, Marcheselli L, et al. Follicular lymphoma international prognostic index 2: a new prognostic index for follicular lymphoma developed by the international follicular lymphoma prognostic factor project. J Clin Oncol. 2009; 27(27):4555 4562.

30. Brice P, Bastion Y, Lepage E, et al. Comparison in low-tumor-burden follicular lymphomas between an initial no-treatment policy, prednimustine, or interferon alfa: a randomized study from the Groupe d'Etude des Lymphomes Folliculaires. Groupe d'Etude des Lymphomes de l'Adulte. J Clin Oncol. 1997;15(3):1110-1117.

31. Cheson BD, Fisher RI, Barrington SF, et al. Recommendations for initial evaluation staging, and response assessment of Hodgkin and non-Hodgkin lymphoma: the Lugano classification. J Clin Oncol. 2014, 32(27):3059-3068
32. Pinnix CC, Ng AK, Dabaja BS, et al. Positron emission tomography-computed tomography predictors of progression after DA-REPOCH for PMBCL. Blood Adv. 2018;2(11): 1334-1343.

33. Barrington SF, Mikhaeel NG, Kostakoglu L, et al. Role of imaging in the staging and response assessment of lymphoma: consensus of the International Conference on Malignant Lymphomas Imaging Working Group. J Clin Oncol. 2014;32(27):3048-3058

34. Falchi L, Keating MJ, Marom EM, et al Correlation between FDG/PET, histology, characteristics, and survival in 332 patients with chronic lymphoid leukemia. Blood. 2014:123(18):2783-2790.

35. Link BK, Maurer MJ, Nowakowski GS, et al. Rates and outcomes of follicular lymphoma transformation in the immunochemotherapy era: a report from the University of Iowa/MayoClinic Specialized Program of Research Excellence Molecular Epidemiology Resource. J Clin Oncol. 2013;31(26):3272-3278.

36. Wagner-Johnston ND, Link BK, Byrtek M, et al. Outcomes of transformed follicular lymphoma in the modern era: a report from the National LymphoCare Study (NLCS). Blood. 2015;126(7):851-857.

37. Alonso-Alvarez S, Magnano L, Alcoceba M, et al. Risk of, and survival following, histological transformation in follicular lymphoma in the rituximab era. A retrospective multicentre study by the Spanish GELTAMO group. Br J Haematol. 2017; 178(5):699708.

38. Gleeson M, Hawkes EA, Peckitt C, et al Outcomes for transformed follicular lymphoma in the rituximab era: the Royal Marsden experience 2003-2013. Leuk Lymphoma. 2017;58(8):1805-1813.

39. Flinn IW, van der Jagt R, Kahl B, et al. Firstline treatment of patients with indolent nonHodgkin lymphoma or mantle-cell lymphoma with bendamustine plus rituximab versus R-CHOP or R-CVP: results of the BRIGHT 5-Year follow-up Study. J Clin Oncol. 2019;37(12):984-991.

40. Casulo C, Burack WR, Friedberg JW. Transformed follicular non-Hodgkin lymphoma. Blood. 2015;125(1):40-47.

41. Nair R, Neelapu SS. The promise of CAR Tcell therapy in aggressive B-cell lymphoma. Best Pract Res Clin Haematol. 2018 31(3):293-298.

42. Locke FL, Ghobadi A, Jacobson CA, et al Long-term safety and activity of axicabtagene ciloleucel in refractory large B-cell lymphoma (ZUMA-1): a single-arm, multicentre, phase 1-2 trial. Lancet Oncol. 2019:20(1):31-42

43. Neelapu SS, Locke FL, Bartlett NL, et al Axicabtagene ciloleucel CAR T-Cell therapy in refractory large B-cell lymphoma. N Engl J Med. 2017;377(26):2531-2544.

44. Schuster SJ, Bishop MR, Tam CS, et al. Tisagenlecleucel in adult relapsed or refractory diffuse large B-Cell lymphoma. N Engl J Med. 2019;380(1):45-56.

45. Kinahan PE, Fletcher JW. Positron emission tomography-computed tomography standardized uptake values in clinical practice and assessing response to therapy. Semin Ultrasound CT MR. 2010;31(6): 496-505.

46. Meignan M, Cottereau AS, Versari A, et al Baseline metabolic tumor volume predicts outcome in high-tumor-burden follicular lymphoma: a pooled analysis of three multicenter studies. J Clin Oncol. 2016; 34(30): 3618-3626. 